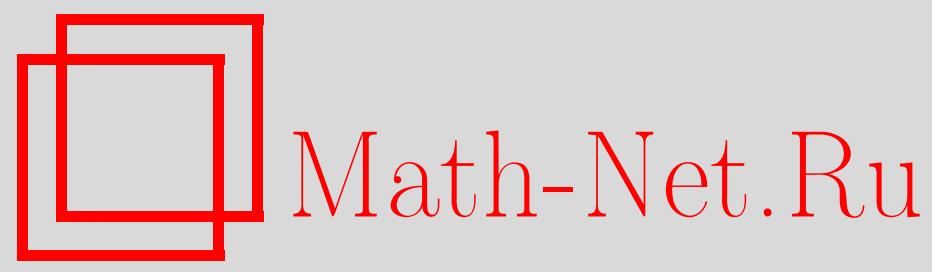

Д. З. Маршан, О перестановках двойной системы Хаара, Матем. заметки, 2000, том 68, выпуск 3, 385-389

DOI: https://doi.org/10.4213/mzm955

Использование Общероссийского математического портала Math-Net.Ru подразумевает, что вы прочитали и согласны с пользовательским соглашением http://www . mathnet.ru/rus/agreement

Параметры загрузки:

IP: 54.197 .217 .227

26 апреля 2023 г., 05:40:11

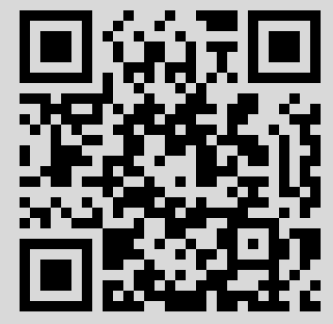




\section{О ПЕРЕСТАНОВКАХ ДВОЙНОЙ СИСТЕМЫ ХААРА}

\section{Д. 3. Маршан}

В работе приводятся оценки нормы оператора перестановки двойной системы Хаара в двоичных пространствах Харди и ВМО.

Библиография: 3 названия.

В настоящей работе приводятся утверждения, связанные с эквивалентностью перестановок двойной системы Хаара в двоичных пространствах Харди и ВМО.

Аналогичные вопросы в одномерном случае изучались в [1], [2] и моей работе [3].

Пусть $\mathbb{N}=\{0,1,2, \ldots\}$,

$$
A_{0}=\left\{\left[k \cdot 2^{-n},(k+1) \cdot 2^{-n}\right): 0 \leqslant k<2^{n}, k, n \in \mathbb{N}\right\},
$$

$A=A_{0} \cup\{[0,1]\}$ и $\chi=\left\{h_{I}, I \in A\right\}$ - система Хаара, занумерованная элементами множества $A$ следующим образом: $h_{[0,1]}(t)=1, t \in[0,1]$, а для $I=\left[k \cdot 2^{-n},(k+1) \cdot 2^{-n}\right) \in A_{0}$

$$
h_{I}(t)= \begin{cases}2^{n / 2} & \text { при } t \in\left[k \cdot 2^{-n},(2 k+1) \cdot 2^{-n-1}\right), \\ -2^{n / 2} & \text { при } t \in\left[(2 k+1) \cdot 2^{-n-1},(k+1) \cdot 2^{-n}\right), \\ 0 & \text { при } t \in[0,1] \backslash I .\end{cases}
$$

Обозначим $A^{(2)}=A \times A=\left\{B=I_{1} \times I_{2}, I_{1}, I_{2} \in A\right\}, A_{0}^{(2)}=A^{(2)} \backslash\{Q\}, Q=[0,1] \times[0,1]$, и пусть $\chi^{(2)}=\left\{h_{B}, B \in A^{(2)}\right\}$ - двойная система Хаара, занумерованная элементами множества $A^{(2)}$ следующим образом:

$$
h_{B}(t)=h_{I_{1}}\left(t_{1}\right) \cdot h_{I_{2}}\left(t_{2}\right), \quad t=\left(t_{1}, t_{2}\right) \in Q,
$$

где $B=I_{1} \times I_{2} \in A^{(2)}, h_{I_{1}}, h_{I_{2}}-$ функции из системы $\chi$.

Пусть $(\mathscr{B}(Q),\|\cdot\|)$ - банахово пространство, в котором линейная оболочка двойной системы Хаaра $\mathscr{L}^{(2)}$ плотна.

Каждая биекция $\mathscr{F}: A^{(2)} \rightarrow A^{(2)}$ порождает на $\mathscr{L}^{(2)}$ оператор

$$
R_{\mathscr{F}}^{(2)} f=\sum_{B \in A^{(2)}} \hat{f}_{B} h_{\mathscr{F}(B)}, \quad \hat{f}_{B}=\int_{Q} f(t) h_{B}(t) d t, \quad B \in A^{(2)}
$$

Если $R_{\mathscr{F}}^{(2)}$ ограничен на $\mathscr{L}^{(2)}$, то его можно продолжить до ограниченного оператора на $\mathscr{B}(Q)$. 
ОПРЕДЕЛЕНИЕ 1. Системы $\chi^{(2)}$ и $\chi_{\mathscr{F}}^{(2)}=\left\{h_{\mathscr{F}(B)}, B \in A^{(2)}\right\}$ назьваются әквивалентны.ми в пространстве $\mathscr{B}(Q)$, если операторы $R_{\mathscr{F}}^{(2)}, R_{\mathscr{F}-1}^{(2)}: \mathscr{B}(Q) \rightarrow \mathscr{B}(Q)$ ограничены и $\mathscr{F}^{-1}$ - отображение, обратное к $\mathscr{F}$.

Пусть $H(Q)$ - двоичное пространство Харди функций двух переменных, т. е.

$$
H(Q)=\left\{f \in L(Q):\|f\|_{H(Q)}=\int_{Q} f^{*}(t) d t<\infty\right\}
$$

где

$$
f^{*}(x)=\sup _{x \in B \in A^{(2)}} \frac{\left|\int_{B} f(t) d t\right|}{|B|}, \quad x=\left(x_{1}, x_{2}\right) \in Q
$$

$|B|$ - мера Лебега множества $B$, и пусть $\mathrm{BMO}(Q)$ - двоичное пространство, сопряженное к $H(Q)$, т. е.

$$
\operatorname{BMO}(Q)=\left\{f \in L(Q): \hat{f}_{Q}=0,\|f\|_{\mathrm{BMO}(Q)}=\sup _{B \in A_{0}^{(2)}}\left(\frac{\sum_{\Pi \subseteq B} \hat{f}_{\Pi}^{2}}{|B|}\right)^{1 / 2}<\infty\right\}
$$

a $\operatorname{VMO}(Q)$ - двоичное пространство, сопряженное к которому есть $H(Q)$, т. е.

$$
\operatorname{VMO}(Q)=\left\{f \in \operatorname{BMO}(Q): \lim _{|B| \rightarrow 0} \frac{\sum_{\Pi \subseteq B} \hat{f}_{\Pi}^{2}}{|B|}=0\right\}
$$

Определим "норму" биекции $\mathscr{F}: A^{(2)} \rightarrow A^{(2)}$

$$
\|\mathscr{F}\|_{2}=\sup _{B \in A_{0}^{(2)}}\left(\frac{\left|\bigcup_{\Pi \subseteq B} \mathscr{F}(\Pi)\right|}{|B|}\right)^{1 / 2}
$$

TЕоремА 1. Пусть $\mathscr{F}: A^{(2)} \rightarrow A^{(2)}-$ произвольная биекиия, $\mathscr{F}(Q)=Q$. Тогда

$$
\left\|R_{\mathscr{F}-1}^{(2)}\right\|_{\mathrm{BMO}(Q) \rightarrow \mathrm{BMO}(Q)} \geqslant\|\mathscr{F}\|_{2}
$$

ДокАЗАТЕЛЬСтво. Возьмем $\varepsilon>0$ и найдем такой элемент $B \in A_{0}^{(2)}$, что

$$
\frac{\left|\bigcup_{\Pi \subseteq B} \mathscr{F}(\Pi)\right|}{|B|}>\left(\|\mathscr{F}\|_{2}-\varepsilon\right)^{2}
$$

Представим множество $\{\mathscr{F}(\Pi): \Pi \subseteq B\}$ в виде последовательности $\left\{\Pi_{k}\right\}_{k=1}^{\infty}$. Определим функцию

$$
\varphi=\left|\Pi_{1}\right|^{1 / 2} h_{\Pi_{1}}+\sum_{n=2}^{\infty}\left(\left|\Pi_{n}\right|-\left|\Pi_{n} \cap\left(\bigcup_{k=1}^{n-1} \Pi_{k}\right)\right|\right)^{1 / 2} h_{\Pi_{n}} .
$$


Замечаем, что $\|\varphi\|_{\mathrm{BMO}(Q)}=1$. Имеем

$$
\begin{aligned}
\frac{\sum_{\Pi \subseteq B} \hat{\varphi}_{\mathscr{F}(\Pi)}^{2}}{|B|} & =\frac{\left|\Pi_{1}\right|+\sum_{n=2}^{\infty}\left(\left|\Pi_{n}\right|-\left|\Pi_{n} \cap\left(\bigcup_{k=1}^{n-1} \Pi_{k}\right)\right|\right)}{|B|} \\
& =\frac{\left|\bigcup_{\Pi \subseteq B} \mathscr{F}(\Pi)\right|}{|B|}>\left(\|\mathscr{F}\|_{2}-\varepsilon\right)^{2} .
\end{aligned}
$$

Поэтому

$$
\left\|R_{\mathscr{F}-1}^{(2)}\right\|_{\mathrm{BMO}(Q) \rightarrow \mathrm{BMO}(Q)} \geqslant\left\|R_{\mathscr{F}-1}^{(2)} \varphi\right\|_{\mathrm{BMO}(Q)}>\|\mathscr{F}\|_{2}-\varepsilon .
$$

В силу произвольности $\varepsilon$ верна оценка (1). Теорема доказана.

Для заданной биекции $\mathscr{F}: A^{(2)} \rightarrow A^{(2)}$ и каждого $B \in A^{(2)}$ пусть $S_{\mathscr{F}}(B)-$ множество, полученное удалением из $\{\mathscr{F}(\Pi): \Pi \subseteq B\}$ всякого прямоугольника, входящего целиком в другой из того же множества.

ОПРЕДЕЛЕниЕ 2. Биекция $\mathscr{F}: A^{(2)} \rightarrow A^{(2)}, \mathscr{F}(Q)=Q$, назьвается слабо пересекающейся, если пересечение любых двух элементов из каждого $S_{\mathscr{F}}(B)$ не содержит других пересечений двух элементов из того же множества.

В частности, к слабо пересекаюшимся биекциям относятся те биекции $\mathscr{F}: A^{(2)} \rightarrow$ $A^{(2)}$, для которых каждое множество $S_{\mathscr{F}}(B)$ состоит из попарно дизъюнктивных прямоугольников.

Теорема 2. Пусть $\mathscr{F}: A^{(2)} \rightarrow A^{(2)}$ - слабо пересекающ,аяся биекиия. Тогда

$$
\left\|R_{\mathscr{F}}^{(2)}\right\|_{H(Q) \rightarrow H(Q)} \sim\left\|R_{\mathscr{F}-1}^{(2)}\right\|_{\mathrm{BMO}(Q) \rightarrow \operatorname{BMO}(Q)} \sim\|\mathscr{F}\|_{2}
$$

СлЕДСТВИЕ 1. Системы $\chi^{(2)} u \chi_{\mathscr{F}}^{(2)}-$ эквивалентные базисы в $H(Q)$ u $\operatorname{VMO}(Q)$, если и только если $\|\mathscr{F}\|_{2}<\infty u\left\|\mathscr{F}^{-1}\right\|_{2}<\infty$.

СледСТвИЕ 2. Пусть $2<p<\infty u q=p /(p-1)$. Тогда

$$
\left\|R_{\mathscr{F}}^{(2)}\right\|_{L^{q}(Q) \rightarrow L^{q}(Q)}=\left\|R_{\mathscr{F}-1}^{(2)}\right\|_{L^{p}(Q) \rightarrow L^{p}(Q)} \sim\|\mathscr{F}\|_{2}^{(p-2) / p} .
$$

СледСтвиЕ 3. Пусть $1<p<\infty$. Системы $\chi^{(2)} u \chi_{\mathscr{F}}^{(2)}-$ әквивалентные базисы в $L^{p}(Q)$, если и только если

$$
\|\mathscr{F}\|_{2}<\infty \quad u \quad\left\|\mathscr{F}^{-1}\right\|_{2}<\infty
$$


ДоКАЗАТЕЛЬСТво тЕОРЕмЫ 2. Сначала докажем неравенство

$$
\left\|R_{\mathscr{F}-1}^{(2)}\right\|_{\mathrm{BMO}(Q) \rightarrow \operatorname{BMO}(Q)} \leqslant \sqrt{2}\|\mathscr{F}\|_{2} .
$$

Пусть $f \in \mathscr{L}^{(2)}$ и $\|f\|_{\mathrm{BMO}(Q)} \leqslant 1$. Имеем

$$
\begin{aligned}
\frac{\sum_{\Pi \subseteq B} \hat{f}_{\mathscr{F}(\Pi)}^{2}}{|B|} & \leqslant \frac{\sum_{\Pi \in S \mathscr{F}(B)} \sum_{K \subseteq \Pi} \hat{f}_{K}^{2}}{|B|} \\
& \leqslant \frac{\sum_{\Pi \in S \mathscr{F}(B)}|\Pi|}{|B|} \leqslant \frac{2\left|\bigcup_{\Pi \subseteq B} \mathscr{F}(\Pi)\right|}{|B|}, \quad B \in A^{(2)}
\end{aligned}
$$

Отсюда следует (4). Неравенства (1) и (4) дают правую оценку в (2).

Оператор $R_{\mathscr{F}}^{(2) *}$, сопряженный к $R_{\mathscr{F}}^{(2)}$, удовлетворяет равенству $R_{\mathscr{F}}^{(2) *}=R_{\mathscr{F}-1}^{(2)}$.

Используя сопряжение, имеем

$$
\left\|R_{\mathscr{F}}^{(2)}\right\|_{H(Q) \rightarrow H(Q)} \sim\left\|R_{\mathscr{F}}^{(2) *}\right\|_{\mathrm{BMO}(Q) \rightarrow \mathrm{BMO}(Q)}=\left\|R_{\mathscr{F}-1}^{(2)}\right\|_{\mathrm{BMO}(Q) \rightarrow \operatorname{BMO}(Q)} \sim\|\mathscr{F}\|_{2}
$$

Теорема доказана.

ДокАЗАТЕЛЬСТвО СЛЕДСТвИЯ 2. Фиксируем $p \in(2, \infty)$. Установим правую оценку в (2).

Возьмем $\varepsilon>0$ и найдем такой элемент $B \in A_{0}^{(2)}$, что

$$
\frac{\left|\bigcup_{\Pi \subseteq B} \mathscr{F}(\Pi)\right|}{|B|}>\left(\|\mathscr{F}\|_{2}-\varepsilon\right)^{2} .
$$

Определим функцию

$$
g=R_{\mathscr{F}-1}^{(2)}\left(\sum_{\Pi \in S_{\mathscr{F}}(B)}|\Pi|^{1 / 2} h_{\Pi}\right) .
$$

Замечаем, что

$$
\left\|R_{\mathscr{F}}^{(2)} g\right\|_{L^{q}(Q)} \geqslant\left|\bigcup_{\Pi \subseteq B} \mathscr{F}(\Pi)\right|^{1 / q}
$$

и

$$
\|g\|_{L^{q}(Q)}^{2} \cdot|B|^{(q-2) / q} \leqslant\|g\|_{L^{2}(Q)}^{2} \leqslant \sum_{\Pi \in S \mathscr{F}(B)}|\Pi| \leqslant 2\left|\bigcup_{\Pi \subseteq B} \mathscr{F}(\Pi)\right| .
$$

Поэтому

$$
\begin{aligned}
\sqrt{2}\left\|R_{\mathscr{F}}^{(2)}\right\|_{L^{q}(Q) \rightarrow L^{q}(Q)} & \geqslant \frac{\sqrt{2}\left\|R_{\mathscr{F}}^{(2)} g\right\|_{L^{q}(Q)}}{\|g\|_{L^{q}(Q)}} \\
& \geqslant\left(\frac{\left|\bigcup_{\Pi \subseteq B} \mathscr{F}(\Pi)\right|}{|B|}\right)^{(2-q) /(2 q)}>\left(\|\mathscr{F}\|_{2}-\varepsilon\right)^{(2-q) / q} .
\end{aligned}
$$


В силу произвольности $\varepsilon$ имеем

$$
\sqrt{2}\left\|R_{\mathscr{F}}^{(2)}\right\|_{L^{q}(Q) \rightarrow L^{q}(Q)} \geqslant\|\mathscr{F}\|_{2}^{(2-q) / q}=\|\mathscr{F}\|_{2}^{(p-2) / p} .
$$

Используя интерполяцию между $H(Q)$ и $L^{2}(Q)$ (см. в одномерном случае [1, с. 140]), убеждаемся в справедливости оценки

$$
\left\|R_{\mathscr{F}}^{(2)}\right\|_{L^{q}(Q) \rightarrow L^{q}(Q)} \leqslant M_{p}\|\mathscr{F}\|_{2}^{(p-2) / p}
$$

где $M_{p}$ - постоянная, зависящая только от $p$. Неравенства (5) и (6) дают правую оценку в (3). Левое равенство в (3) очевидно. Следствие доказано.

Другие следствия очевидньм образом вытекают соответственно из теоремы 2 и следствия 2.

\section{СПИСОК ЦИТИРОВАННОЙ ЛИТЕРАТУРЫ}

[1] Schipp F. On equivalence of rearrangements of the Haar system in dyadic Hardy and BMO spaces // Analysis Math. 1990. V. 16. № 2. P. 135-141.

[2] Семёнов Е. М., Штекерт Б. Перестановки системы Хаара в пространствах $L^{p} / /$ Analysis Math. 1981. V. 7. № 4. P. 277-295.

[3] Отырба Д. З. О перестановках в пространстве ВМО системы Хаара // УМН. 1994. Т. 49. №3. C. $183-184$.

Абхазский государственный университет 